Wiener klinische Wochenschrift The Central European Journal of Medicine

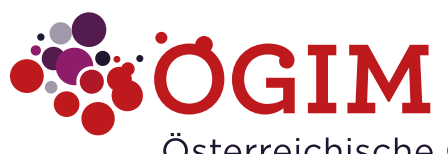

Österreichische Gesellschaft für Innere Medizin

\section{Vorstand und Ausschuss ab 1. Jänner 2013}

\author{
Vorsitzender \\ Univ.Prof.Dr. Herbert Watzke \\ Stellvertretender Vorsitzender \\ Univ.Prof.Dr. Ernst Pilger \\ Generalsekretär \\ Ao.Univ.Prof.Dr. Markus Peck-Radosavljevic \\ Kassier \\ Prim.Univ.Doz.Dr. Franz Xaver Roithinger
}

Fachärzteausbildungsverantwortlicher

Doz.Dr. Bernhard Angermayr

Bundesfachgruppenobmann für Innere Medizin in der ÖÄK (ex offo)

Dr. Lothar Fiedler

\author{
Vorstände der Univ.Kliniken für Innere Medizin \\ (ex offo) \\ o.Univ.Prof.Dr. Gerald Maurer \\ o.Univ.Prof.Dr. Otmar Pachinger \\ Univ.Prof.Dr. Ernst Pilger \\ o.Univ.Prof.Dr. Josef Smolen \\ Univ.Prof.Dr. Christoph Zielinski
}

Tagungspräsident 2013

Prim.o.Univ.Prof.DDr.h.c. Heinz Drexel

Tagungssekretär 2013

Priv.Doz.Dr. Christoph Säly

Präsidenten der wissenschaftlichen Gesellschaften der internistischen Additivfächer (ex offo)

Ao.Univ.Prof. DI Dr. Sabina Baumgartner-Parzer - Österreichische Gesellschaft für Endokrinologie und Stoffwechsel

Prim.Univ.Prof.Dr. Karl Benedetto - Österreichische Gesellschaft für Sportmedizin und Prävention

Ao.Univ.Prof.Dr. Marianne Brodmann - Österreichische Gesellschaft für internistische Angiologie

Prim.Univ.Prof.Dr. Günther Gastl - Österreichische Gesellschaft für Hämatologie und Onkologie

Ao.Univ.Prof.DDr. Manfred Herold - Österreichische Gesellschaft für Rheumatologie und Rehabilitation
Ao.Univ.Prof.Dr. Bernd Jilma - Österreichische Pharmakologische Gesellschaft

o.Univ.Prof.Dr. Michael Joannidis - Österreichische Gesellschaft für internistische und allgemeine Intensivmedizin und Notfallmedizin

Univ.Prof.Dr. Wolfgang Petritsch - Österreichische Gesellschaft für Gastroenterologie und Hepathologie

Univ.Prof.Dr. Burkert Pieske - Österreichische Kardiologische Gesellschaft

Prim.Dr. Katharina Pils - Österreichische Gesellschaft für Geriatrie und Gerontologie

Prim.Univ.Prof.Dr. Erich Pohanka - Österreichische Gesellschaft für Nephrologie

Univ.Prof.Dr. Günter Weiss - Österreichische Gesellschaft für Infektionskrankheiten und Tropenmedizin

Präsident der Österreichischen Gesellschaft für Pneumologie (ex offo)

OA Dr. Sylvia Hartl

Fortbildungsverantwortlicher

Ao.Univ.Prof.Dr. Florian Thalhammer

Gewählte Ausschussmitglieder

Prim.Univ.Prof.Dr. Meinhard Kneussl

Prim.Prof.Dr. Kurt Lenz

Chefärztin Prof.Dr. Petra-Maria Schumm-Dräger

Dr. Günther Wawrowsky

Regionale Fortbildungsbeauftragte (ex offo)

Univ.Prof.Dr. Renate Koppensteiner

o.Univ.Prof.Dr. Otmar Pachinger

Prim.Univ.Prof.Dr. Bruno Schneeweiss

Univ.Prof.Ing.Dr. Gerhard Stark

Priv.Doz. OA Dr. Gernot Wolkersdörfer 